\title{
Sicherheit von Studienpatienten muss an erster Stelle stehen
}

Newell KA, Metha AK, Larsen CP et al. Lessons learned: Early termination of a randomized trial of calcineurin inhibitor and corticosteroid avoidance using betalacept. Am J Transplant 2017; May 28; doi: 10.1111/ajt.14377 [Epub ahead of print]

Durch eine Gabe von Betalacept das langfristige Transplantatüberleben zu verbessern, schwerwiegende Nebenwirkungen der immunsuppression zu reduzieren und so die Immunsuppressive Therapie nach einer Nierentranpslantation zu optimieren, war Ziel der vorliegenden Studie. Aufgrund einer vergleichsweise hohe Rate an zellulären Abstoßungsreaktionen und einer prothrombotischen Komponente bei den Infusionen von 2 Proteinkomponenten unter der Belatacepttherapie musste diese Studie im Interesse der Studienteilnehmer allerdings abgebrochen werden.

Aufgrund vielzähliger Bemühungen konnten in den letzten Jahren insbesondere das kurzfristige Transplantatüberleben und die Anzahl akuter Abstoßungen nach der Nierentransplantation weiter verbessert werden. Allerdings führten im Langzeitverlauf ein hohes Spender- und Empfängeralter, eine höhere medikamentöse Komplexität und Langzeitnebenwirkungen sowie eine fehlende Adhärenz der Patienten häufig zu einem vorzeitigen Transplantatverlust.

Ziel der vorliegenden Studie war, in der Frühphase nach der Nierentransplantation die medikamentöse immunsuppressive Therapie durch die Gabe eines CTLA-4-Blockers (CTLA-4 = „cytotoxic T-lymphocyteassociated protein 4“) zu optimieren, um eine calcineurininibitor- und steroidfreie Therapie zu erreichen. Hierdurch erhoffte man sich positive Effekte - zum einen auf die Nephrotoxizität der Behandlung, aber auch auf Hochdruckerkrankungen, die Neuentstehung eines Diabetes, Hyperlipidämien und die daraus resultierenden Spätfolgen der damit assoziierten Herz-Kreislauf- und zerebrovaskulären Erkrankungen nach der Transplantation.

Es wurden 3 Patientengruppen entweder mit Tacrolimus/Alemtuzumab (Gruppe 1), Belatacept/Alemtuzumab (Gruppe 2) und Belatacept/Tacrolimus/Basiliximab (Gruppe 3 ) therapiert. Eingeschlossen in die Studie wurden 19 Patienten, 68,4\% waren Nieren-
Lebendspende-Empfänger. Die mittlere Nachbeobachtungszeit betrug 1091 Tage.

Insgesamt kam es zu 5 Transplantatverlusten, davon 2 in Gruppe 1 und zwar einer aufgrund einer Staphylokokkensepsis und ein weiterer nach einem Jahr aufgrund einer Glomerulopathie. Die 3 Transplantatverluste in Gruppe 2 wurden Gefäßthrombosen und einem technischen Fehler zugeordnet. 7 Patienten aus den Gruppen 2 und 3 (53,8\%) erlitten eine Rejektion, die Nierenfunktion war mit einer glomerulären Filtrationsrate (GFR) zwischen 51,6 und $58,3 \mathrm{ml} / \mathrm{min}$ in allen 3 Gruppen exzellent und ohne signifikanten Unterschied.

Aufgrund der hohen Anzahl von Transplantatverlusten wurde die Studie mit dem Hinweis auf schwerwiegende Nebenwirkungen (SAEs: „severe adverse events“) unterbrochen. Dieses Vorgehen unterstreicht die Verantwortung der Studienleiter gegenüber den Studienteilnehmern im Interesse der Abwägung von Risiken und Vorteilen einer experimentellen Therapie.

\section{Kommentar}

In den letzten Jahren wurde bei der Immunsuppression nach einer Nierentransplantation verstärkt auf die Langzeitfolgen der etablierten Immunsuppressiva geachtet und eine rasche Reduktion von Calcineurininhibitoren und Steroiden oder auch deren Vermeidung propagiert. Mit der Einführung von Belatacept im Jahr 2011 zeichnete sich ein Vorteil hinsichtlich der Nierenfunktion im Langzeitverlauf ab. In Studien zeigten sich jedoch häufiger Rejektionen gegenüber der konventionellen Therapie bei einer allerdings selteneren Bildung von Anti-HLA-Antikörpern (HLA: humanes Leukozytenantigen). Insgesamt konnte in älteren Studien keine eindeutige Überlegenheit von Belatacept festgestellt werden.

Dieser aktuelle Versuch, die Effektivität von Belatacept im Vergleich mit der Therapie mit Calcineurininhibitoren erneut zu untersuchen, wurde leider durch die hohe
Anzahl an Tranplantatverlusten überschattet. Daher wurde die Studie abgebrochen.

Auch wenn die Transplantatverluste zum Teil technischen Fehlern anzulasten sein mögen, muss die prothrombotische Komponente bei Infusionen von 2 Proteinkomponenten diskutiert werden. Ebenso war eine höhere Rate an akuten zellulären Abstoßungen in der Belataceptgruppe zu konstatieren. CD4-Gedächniszellen, die bereits vor der Transplantation zu finden sind, scheinen mit einem erhöhten Risiko für eine Rejektion bei der Behandlung mit Belatacept assoziiert zu sein.

Insgesamt ist die Durchführung einer solchen Studie zur Verbesserung der Nierenlangzeitfunktion mit einer Verminderung der durch Calcineurininhibitoren verbundenen Spätfolgen zweifellos wichtig und wünschenswert. Andererseits dürfen die Teilnehmer solcher Untersuchungen keinesfalls gefährdet werden. Daher ist eine Unterbrechung der Studie im Interesse der Studienteilnehmer und im Rahmen der Verantwortung der Studienleiter unabdingbar.

\section{Korrespondenzadresse}

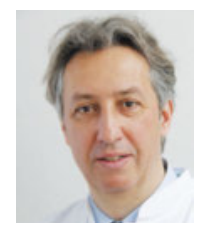

Prof. Dr. med. Stefan Thorban

Transplantationszentrum Klinik und Poliklinik für Chirurgie

Klinikum rechts der Isar Technische Universität München Ismaninger Straße 22 81675 München stefan.thorban@tum.de

Literatur

[1] Vincenti F, Rostaing L, Grinyo J et al. Belatacept and long-term outcomes in kidney transplantation. N Engl J Med 2016; 374: 333-343; doi: 10.1056/NEJMoa1506027

[2] Kirk AD, Guasch A, Xu H et al. Renal transplantation using belatacept without maintenance steroids or calcineurin inhibitors. Am J Transplant 2014; 14: 1142-1151; doi: 10.1111/ajt.12712 